Indian J. Pure Appl. Math., 51(2): 611-630, June 2020

(c) Indian National Science Academy

DOI: $10.1007 / \mathrm{s} 13226-020-0420-\mathrm{X}$

\title{
MULTIPLE POSITIVE SOLUTIONS FOR SINGULAR ELLIPTIC PROBLEMS INVOLVING CONCAVE-CONVEX NONLINEARITIES AND SIGN-CHANGING POTENTIAL ${ }^{1}$
}

\author{
Hong-Ying $\mathrm{Li}^{*}$, Yang $\mathrm{Pu}^{*}$ and Jia-Feng Liao** \\ *School of Mathematics and Information, China West Normal University, \\ Nanchong Sichuan 637002, People's Republic of China \\ ${ }^{* *}$ College of Mathematics Education, China West Normal University, \\ Nanchong Sichuan 637002, People's Republic of China \\ e-mails: lihongyingnch@163.com; 172431794@qq.com; liaojiafeng@163.com
}

(Received 9 November 2018; accepted 23 March 2019)

In this paper, we are interested in considering the following singular elliptic problem with concaveconvex nonlinearities

$$
\begin{cases}-\Delta u-\frac{\mu}{|x|^{2}} u=f(x)|u|^{p-2} u+g(x)|u|^{q-2} u, & \text { in } \Omega \backslash\{0\} \\ u=0, & \text { on } \partial \Omega\end{cases}
$$

where $\Omega \subset \mathbb{R}^{N}(N \geq 3)$ is a smooth bounded domain with $0 \in \Omega, 0<\mu<\bar{\mu}=\frac{(N-2)^{2}}{4}, 1<$ $q<2<p<2^{*}$ and $2^{*}=\frac{2 N}{N-2}$ is the Sobolev critical exponent, the coefficient functions $f, g$ may change sign on $\Omega$. By the Nehari method, we obtain two solutions, and one of them is a ground state solution. Under some stronger conditions, we point that the two solutions are positive solutions by the strong maximum principle.

Key words : Singular elliptic problem; concave-convex nonlinearities; ground state solution; Nehari method.

2010 Mathematics Subject Classification : 35J20, 35J61, 35D30.

\footnotetext{
${ }^{1}$ The paper is supported by the Scientific Research Fund of Sichuan Provincial Education Department(18ZA0471), the Meritocracy Research Funds of China West Normal University (17YC383), the Fundamental Research Funds of China West Normal University (17E089, 18B015, 18D052) and the Innovation Team Research Funds of China West Normal University (CXTD2018-8).
} 


\section{IntRoduction AND MAIn RESUlts}

Consider the following singular elliptic problem with concave-convex nonlinearities

$$
\begin{cases}-\Delta u-\frac{\mu}{|x|^{2}} u=f(x)|u|^{p-2} u+g(x)|u|^{q-2} u, & \text { in } \Omega \backslash\{0\} \\ u=0, & \text { on } \partial \Omega\end{cases}
$$

where $\Omega \subset \mathbb{R}^{N}(N \geq 3)$ is a smooth bounded domain with $0 \in \Omega, 0<\mu<\bar{\mu}=\frac{(N-2)^{2}}{4}, 1<$ $q<2<p<2^{*}$ and $2^{*}=\frac{2 N}{N-2}$ is the Sobolev critical exponent. The coefficient functions $f \in$ $L^{\frac{2^{*}}{2^{*}-p}}(\Omega), g \in L^{\frac{2^{*}}{2^{*}-q}}(\Omega)$ and satisfy the following condition.

$\left(H_{0}\right)$ The sets $\{x \in \Omega: f(x)>0\}$ and $\{x \in \Omega: g(x)>0\}$ with positive measures, that is, $f, g \geq 0$ or $f, g$ change sign on $\Omega$.

Very recently, Chen and Chen studied problem (1.1) with $f(x)=\mu W(x)$, where $W, g \in C(\bar{\Omega})$, $g(x) \geq 0$ and the set $\{x \in \Omega: W(x)>0\}$ with positive measure, see [10]. By the Nehari method, they obtained that there exists $T_{\lambda}>0$ such that problem (1.1) has at least two positive solutions for $0<\mu<T_{\lambda}$.

When $\mu=0$ in problem (1.1), it reduces to the classic semilinear elliptic problem with concaveconvex nonlinearities. Much interest has grown on the concave-convex problem, starting from the celebrated paper of Ambrosetti, Brézis and Cerami [1]. After that, the concave-convex problem has been extensively studied, and some important and interesting results have been obtained, such as [2-4, 6-12, 14, 17-24, 26-32]. Particularly, Sun [26] considered problem (1.1) with $\lambda=0, f, g$ satisfying the same conditions of [10]. By using the Nehari method, the author obtained that there exists $T_{\lambda}>0$ such that problem (1.1) has at least two positive solutions for $0<\lambda<T_{\lambda}$. In fact, [10] generalized the results of [26] to problem (1.1).

In this article, we are interested in studying multiple solutions of problem (1.1). Motivated by [10] and [26], an interesting study is the relevance of coefficient functions of the concave-convex terms to the multiplicity of solutions of problem (1.1). In the present paper, by the Nehari method, we get two nonnegative solutions of problem (1.1) under some constraint conditions on $f, g$; then, we obtain two positive solutions of problem (1.1) under some stronger constraint conditions on $f, g$.

Let $S_{\mu}$ be the best Sobolev constant, that is

$$
S_{\mu}:=\inf _{u \in D^{1,2}\left(\mathbb{R}^{N}\right) \backslash\{0\}} \frac{\int_{\mathbb{R}^{N}}\left(|\nabla u|^{2}-\frac{\mu}{|x|^{2}} u^{2}\right) d x}{\left(\int_{\mathbb{R}^{N}}|u|^{2^{*}} d x\right)^{\frac{2}{2^{*}}}}=\inf _{u \in H_{0}^{1}(\Omega) \backslash\{0\}} \frac{\int_{\Omega}\left(|\nabla u|^{2}-\frac{\mu}{|x|^{2}} u^{2}\right) d x}{\left(\int_{\Omega}|u|^{2^{*}} d x\right)^{\frac{2}{2^{*}}}} .
$$


The energy functional corresponding to problem (1.1) is defined as follows

$$
I(u)=\int_{\Omega}\left(|\nabla u|^{2}-\frac{\mu}{|x|^{2}} u^{2}\right) d x-\frac{1}{p} \int_{\Omega} f(x)|u|^{p} d x-\frac{1}{q} \int_{\Omega} g(x)|u|^{q} d x
$$

for all $u \in H_{0}^{1}(\Omega)$. We denote $\|u\|_{\mu}=\left[\int_{\Omega}\left(|\nabla u|^{2}-\frac{\mu}{|x|^{2}} u^{2}\right) d x\right]^{\frac{1}{2}}$ as the norm of $H_{0}^{1}(\Omega)$. By the the Hardy inequality (see [5] or [15]), one has

$$
\int_{\Omega} \frac{\mu}{|x|^{2}} u^{2} d x \leq \frac{1}{\bar{\mu}} \int_{\Omega}|\nabla u|^{2} d x
$$

for all $u \in H_{0}^{1}(\Omega)$. Thus, the norm $\|u\|_{\mu}$ is equivalent to the usual norm $\|u\|=\left(\int_{\Omega}|\nabla u|^{2} d x\right)^{\frac{1}{2}}$ of $H_{0}^{1}(\Omega)$.

In general, a function $u$ is called a weak solution of problem (1.1) if $u \in H_{0}^{1}(\Omega)$ and for all $\varphi \in H_{0}^{1}(\Omega)$ it holds

$$
\int_{\Omega}\left[(\nabla u, \nabla \varphi)-\frac{\mu}{|x|^{2}} u \varphi\right] d x-\int_{\Omega} f(x)|u|^{p-2} u \varphi d x-\int_{\Omega} g(x)|u|^{q-2} u \varphi d x=0 .
$$

So if such a solution exists then it must lie in Nehari manifold $\mathscr{N}$, which is defined by

$$
\mathscr{N}=\left\{u \in H_{0}^{1}(\Omega):\|u\|_{\mu}^{2}-\int_{\Omega} f(x)|u|^{p} d x-\int_{\Omega} g(x)|u|^{q} d x=0\right\} .
$$

In order to obtain the multiplicity of solutions, we make splitting for $\mathscr{N}$. For this purpose, we define a fibering map $J_{u}: t \rightarrow I(t u)$ for all $t>0$, that is,

$$
J_{u}(t)=\frac{t^{2}}{2}\|u\|_{\mu}^{2}-\frac{t^{p}}{p} \int_{\Omega} f(x)|u|^{p} d x-\frac{t^{q}}{q} \int_{\Omega} g(x)|u|^{q} d x
$$

where $u \in H_{0}^{1}(\Omega)$. Then we have

$$
\begin{gathered}
J_{u}^{\prime}(t)=t\|u\|_{\mu}^{2}-t^{p-1} \int_{\Omega} f(x)|u|^{p} d x-t^{q-1} \int_{\Omega} g(x)|u|^{q} d x, \\
J_{u}^{\prime \prime}(t)=\|u\|_{\mu}^{2}-(p-1) t^{p-2} \int_{\Omega} f(x)|u|^{p} d x-(q-1) t^{q-2} \int_{\Omega} g(x)|u|^{q} d x .
\end{gathered}
$$

Clearly, $\mathscr{N}=\left\{u \in H_{0}^{1}(\Omega): J_{u}^{\prime}(1)=0.\right\}$. For all $u \in \mathscr{N}$,

$$
\begin{aligned}
J_{u}^{\prime \prime}(1) & =\|u\|_{\mu}^{2}-(p-1) \int_{\Omega} f(x)|u|^{p} d x-(q-1) \int_{\Omega} g(x)|u|^{q} d x \\
& =(2-q) \int_{\Omega} g(x)|u|^{q} d x-(p-2) \int_{\Omega} f(x)|u|^{p} d x \\
& =(2-p)\|u\|_{\mu}^{2}+(p-q) \int_{\Omega} g(x)|u|^{q} d x \\
& =(2-q)\|u\|_{\mu}^{2}-(p-q) \int_{\Omega} f(x)|u|^{p} d x .
\end{aligned}
$$


Thus, we make the following decomposition

$$
\begin{aligned}
& \mathscr{N}^{+}=\left\{u \in \mathscr{N}: J_{u}^{\prime \prime}(1)>0\right\}, \\
& \mathscr{N}^{0}=\left\{u \in \mathscr{N}: J_{u}^{\prime \prime}(1)=0\right\}, \\
& \mathscr{N}^{-}=\left\{u \in \mathscr{N}: J_{u}^{\prime \prime}(1)<0\right\} .
\end{aligned}
$$

Our approach to problem (1.1) is upon the structure of the constrained sets $\mathscr{N}^{ \pm}, \mathscr{N}^{0}$. Under some conditions, we can prove that $\mathscr{N}^{ \pm} \neq \emptyset$ and $\mathscr{N}^{0}=\{0\}$.

The main results can be described as follows:

Theorem 1.1 - Assume that $0<\mu<\bar{\mu}=\frac{(N-2)^{2}}{4}, 1<q<2<p<2^{*}$ and $f \in L^{\frac{2^{*}}{2^{*}-p}}(\Omega), g \in$ $L^{\frac{2^{*}}{2^{*}-q}}(\Omega)$ satisfy $\left(H_{0}\right)$. Then there exists $\Lambda>0$ such that problem (1.1) has at least two nonnegative solutions for all $|f|_{\frac{2^{*}}{2^{*}-p}}^{\frac{1}{p-2}}|g|_{\frac{2^{*}}{2^{*}-q}}^{\frac{1}{2-q}}<\Lambda$, and one of them is a ground state solution.

Remark 1.1 : To our best knowledge, our result is up to now. Our Theorem 1.1 is a general result of the multiplicity of nonnegative solutions of the singular elliptic problem involving concave-convex nonlinearities. And, we should point out that $f, g$ are may change sign in $\Omega$. Moreover, when $\mu \equiv 0$, Theorem 1.1 is also true.

Theorem 1.2 - Suppose $\Omega \subset \mathbb{R}^{N}(N \geq 3)$ is a bounded domain with smooth boundary $\partial \Omega$. Assume that $0<\mu<\bar{\mu}=\frac{(N-2)^{2}}{4}, 1<q<2<p<2^{*}$ and $f, g$ satisfy the following condition,

$\left(H_{1}\right) f \in L^{\infty}(\Omega)$ with the set $\{x \in \Omega: f(x)>0\}$ of positive measures, and $g \in L^{\infty}(\Omega)$ with $g(x) \geq 0, g \not \equiv 0$.

Then the same conclusions of Theorem 1.1 hold. Moreover, the two nonnegative solutions are positive solutions.

Remark 1.2 : Under the condition of $\left(H_{1}\right)$, by the strong maximum principle, we can prove that the nonnegative solutions are positive. According to [16] (pp: 158, 198), the condition of the boundary of $\Omega$ is necessary. Compared with Theorem 1.1 in [10], our Theorem 1.2 is more general. Particularly, when $\mu \equiv 0$, Theorem 1.2 is also true which generalizes Theorem 1.1 in [26].

Theorem 1.3 - Assume that $0<\mu<\bar{\mu}=\frac{(N-2)^{2}}{4}, 1<q<2<p<2^{*}$ and f,g satisfy the following condition,

$\left(H_{2}\right) f \in L^{\frac{2^{*}}{2^{*}-p}}(\Omega), g \in L^{\frac{2^{*}}{2^{*}-q}}(\Omega)$ are nonzero and nonnegative functions. 
Then the same conclusions of Theorem 1.1 hold. Moreover, the two nonnegative solutions are positive solutions, and the positive ground state solution belongs to $\mathscr{N}^{+}$.

Remark 1.3 : It is worth noticing that we could not confirm the ground state solution lying in $\mathscr{N}^{+}$ or $\mathscr{N}^{-}$when $f$ may change sign, because $\mathscr{N}^{+}$and $\mathscr{N}^{-}$may not be connected submanifolds. Under the condition of $\left(H_{2}\right)$, we obtain that the positive ground state solution lies in $\mathscr{N}^{+}$.

This paper is organized as following. We present some preliminary results in Section 2. In Section 3, we give the proof of Theorems 1.1-1.3. Throughout this paper, the norm in $L^{p}(\Omega)$ is denoted by $|u|_{p}=\left(\int_{\Omega}|u|^{p} d x\right)^{\frac{1}{p}}$.

\section{Some PRELIMINARY ReSUlts}

In this section, we give some lemmas in preparation for the proof of our main results.

Lemma 2.1 - Assume that $f \in L^{\frac{2^{*}}{2^{*}-p}}(\Omega), g \in L^{\frac{2^{*}}{2^{*}-q}}(\Omega)$ satisfy $\left(H_{0}\right)$, then there exists a constant $\Lambda>0$ such that $\mathscr{N}^{ \pm} \neq \emptyset$ for $|f|_{\frac{2^{*}}{2^{*}-p}}^{\frac{1}{p-2}}|g|_{\frac{2^{*}-q}{2-q}}^{\frac{1}{2^{*}-q}}<\Lambda$. Moreover, $\mathscr{N}^{0}=\{0\}$ and $\mathscr{N}^{-}$is a closed set for $|f|_{\frac{2^{*}}{2^{*}-p}}^{\frac{1}{p-2}}|g|_{\frac{2^{*}}{2^{*}-q}}^{\frac{1}{2-q}}<\Lambda$.

PROOF : According to the assumptions of $f$ there exists $u \in H_{0}^{1}(\Omega)$ such that

$$
\int_{\Omega} f(x)|u|^{p} d x>0 .
$$

In fact, let $E=\{x \in \Omega: f(x)>0\}$, one obtains that $E$ is a positive measure set. Then for any $\varepsilon>0$ there exist a closed set $F$ and a open set $G$ such that $F \subset E \subset G$ and mes $(G-F)<\varepsilon$. From the arbitrariness of $\varepsilon$, we have mes $F>0$. We choose $\tilde{u} \in C_{0}^{1}(\Omega)$ with $0 \leq \tilde{u} \leq 1$ such that $\tilde{u}=1$ in $F$ and $\tilde{u}=0$ in $\Omega \backslash G$. Obviously, $\tilde{u} \in H_{0}^{1}(\Omega)$. By Hölder's inequality, one has

$$
\begin{aligned}
\int_{\Omega} f(x)|\tilde{u}|^{p} d x & \geq \int_{F} f(x) d x-\int_{G-F}|f(x) \| \tilde{u}|^{p} d x \\
& \geq \int_{F} f(x) d x-(\operatorname{mes}(G-F))^{\frac{p}{2^{*}}}\left(\int_{G-F}|f|^{\frac{2^{*}}{2^{*}-p}} d x\right)^{\frac{2^{*}-p}{2^{*}}} \\
& \geq \int_{F} f(x) d x-\varepsilon^{\frac{p}{2^{*}}}|f|_{\frac{2^{*}}{2^{*}-p}} \\
& \geq \frac{1}{2} \int_{F} f(x) d x \\
& >0
\end{aligned}
$$

where we choose $\varepsilon=\min \left\{\left(\frac{\int_{F} f(x) d x}{2|f| \frac{2^{*}}{2^{*}-p}}\right)^{\frac{2^{*}}{p}}, \frac{\text { mes } G}{2}\right\}$ such that mes $F \geq \frac{\text { mes } G}{2}>0$ and $\varepsilon^{\frac{p}{2^{*}}}|f|_{\frac{2^{*}}{2^{*}-p}} \leq$ $\frac{1}{2} \int_{F} f(x) d x$. Similarly, we can prove that there exists $u \in H_{0}^{1}(\Omega)$ such that $\int_{\Omega} g(x)|u|^{q} d x>0$. 
Case $A$ : For any $u \in H_{0}^{1}(\Omega)$ such that $\int_{\Omega} g(x)|u|^{q} d x>0$, we define $\Phi \in C\left(\mathbb{R}^{+}, \mathbb{R}\right)$ by

$$
\Phi(t)=t^{2-p}\|u\|_{\mu}^{2}-t^{q-p} \int_{\Omega} g(x)|u|^{q} d x .
$$

Then,

$$
\Phi^{\prime}(t)=-(p-2) t^{1-p}\|u\|_{\mu}^{2}+(p-q) t^{q-p-1} \int_{\Omega} g(x)|u|^{q} d x,
$$

let $\Phi^{\prime}(t)=0$, we can verify

$$
t_{\max }=\left[\frac{(p-q) \int_{\Omega} g(x)|u|^{q} d x}{(p-2)\|u\|_{\mu}^{2}}\right]^{\frac{1}{2-q}} .
$$

Simple computations show that $\Phi^{\prime}(t)>0$ for all $0<t<t_{\max }$ and $\Phi^{\prime}(t)<0$ for all $t>t_{\max }$. Thus $\Phi(t)$ attains its maximum at $t_{\max }$, that is,

$$
\Phi\left(t_{\max }\right)=\frac{2-q}{p-2}\left[\frac{(p-2)}{p-q}\right]^{\frac{p-q}{2-q}} \frac{\|u\|_{\mu}^{\frac{2(p-q)}{2-q}}}{\left(\int_{\Omega} g(x)|u|^{q} d x\right)^{\frac{p-2}{2-q}}} .
$$

From (1.2), we have

$$
S_{\mu}|u|_{2^{*}}^{2}<\|u\|_{\mu}^{2}
$$

and by Hölder's inequality, one has

$$
\begin{aligned}
& \int_{\Omega} f(x)|u|^{p} d x \leq|f|_{\frac{2^{*}}{2^{*}-p}}|u|_{2^{*}}^{p}, \\
& \int_{\Omega} g(x)|u|^{q} d x \leq|g|_{\frac{2^{*}}{2^{*}-q}}|u|_{2^{*}}^{q} .
\end{aligned}
$$

Then from (2.1)-(2.3), one gets

$$
\begin{aligned}
\Phi\left(t_{\text {max }}\right)-\int_{\Omega} f(x)|u|^{p} d x> & \left.\frac{2-q}{p-2}\left(\frac{p-2}{p-q}\right)^{\frac{p-q}{2-q}} \frac{\|u\|_{\mu}^{\frac{2(p-q)}{2-q}}}{\left(|u|_{2^{*}}^{q}|g|_{\frac{2^{*}}{2^{*}-q}}\right.}\right)^{\frac{p-2}{2-q}} \\
& -|f|_{\frac{2^{*}}{2^{*}-p}}|u|_{2^{*}}^{p} \\
= & \left\{\frac{2-q}{p-2}\left(\frac{p-2}{p-q}\right)^{\frac{p-q}{2-q}}\left(\frac{\|u\|_{\mu}^{2}}{|u|_{2^{*}}^{2}}\right)^{\frac{p-q}{2-q}} \frac{1}{|f|_{\frac{2^{*}}{2^{*}-p}}|g|_{\frac{2^{*}}{2^{*}-q}}^{\frac{p-2}{2}}}\right. \\
> & -1\}|f|_{\frac{2^{*}}{2^{*}-p}}|u|_{2^{*}}^{p}
\end{aligned}
$$

when $|f|_{\frac{2^{*}}{2^{*}-p}}^{\frac{1}{p-2}}|g|_{\frac{2^{*}}{2^{*}-q}}^{\frac{1}{2-q}}<\Lambda$, where we can choose $\Lambda=\left(\frac{2-q}{p-q}\right)^{\frac{1}{p-2}}\left(\frac{p-2}{p-q}\right)^{\frac{1}{2-q}} S_{\mu}^{\frac{p-q}{(p-2)(2-q)}}$. 
On the one hand, when $\int_{\Omega} f(x)|u|^{p} d x \leq 0$. Since $\Phi(t) \rightarrow-\infty$ as $t \rightarrow 0^{+}$and $\Phi(t) \rightarrow 0$ as $t \rightarrow+\infty$, there exists a unique $t^{+}$satisfying $0<t^{+}<t_{\max }$ such that

$$
\int_{\Omega} f(x)|u|^{p} d x=\Phi\left(t^{+}\right), \quad \Phi^{\prime}\left(t^{+}\right)>0,
$$

that is, $t^{+} u \in \mathscr{N}^{+}$. Moreover, since

$$
\begin{gathered}
J_{u}(t)=I(t u)=\frac{t^{2}}{2}\|u\|_{\mu}^{2}-\frac{t^{p}}{p} \int_{\Omega} f(x)|u|^{p} d x-\frac{t^{q}}{q} \int_{\Omega} g(x)|u|^{q} d x, \\
J_{u}^{\prime}(t)=\frac{d I(t u)}{d t}=t^{p-1}\left[\Phi(t)-\int_{\Omega} f(x)|u|^{p} d x\right]
\end{gathered}
$$

one gets $\frac{d I(t u)}{d t}<0$ for all $0 \leq t<t^{+}$, and $\frac{d I(t u)}{d t}>0$ for all $t>t^{+}$. Thus

$$
I\left(t^{+} u\right)=\inf _{0 \leq t \leq t_{\max }} I(t u)
$$

On the other hand, when $\int_{\Omega} f(x)|u|^{p} d x>0$, there exist $t_{0}^{+}$and $t_{0}^{-}$satisfying $0<t_{0}^{+}<t_{\max }<t_{0}^{-}$ such that

$$
\Phi\left(t_{0}^{+}\right)=\int_{\Omega} f(x)|u|^{p} d x=\Phi\left(t_{0}^{-}\right)
$$

and

$$
\Phi^{\prime}\left(t_{0}^{-}\right)<0<\Phi^{\prime}\left(t_{0}^{+}\right),
$$

that is, $t_{0}^{+} u \in \mathscr{N}^{+}$and $t_{0}^{-} u \in \mathscr{N}^{-}$. Moreover, according to (2.5) and (2.6), we have $\frac{d I(t u)}{d t}<0$ for all $0 \leq t<t_{0}^{+}$and $\frac{d I(t u)}{d t}>0$ for all $t_{0}^{+}<t<t_{0}^{-}$, and $\frac{d I(t u)}{d t}<0$ for all $t>t_{0}^{-}$. Consequently, one has

$$
I\left(t_{0}^{+} u\right)=\inf _{0 \leq t<t_{\max }} I(t u), \quad I\left(t_{0}^{-} u\right)=\sup _{t \geq t_{\max }} I(t u)
$$

Thus $\mathscr{N}^{ \pm}$are non-empty whenever $|f|_{\frac{2^{*}}{2^{*}-p}}^{\frac{1}{p-2}}|g|_{\frac{2^{*}}{2^{*}-q}}^{\frac{1}{2-q}}<\Lambda$.

Next, we prove that $\mathscr{N}^{0}=\{0\}$ for $|f|_{\frac{2^{*}}{2^{*}-p}}^{\frac{1}{p-2}}|g|_{\frac{2^{*}}{2^{*}-q}}^{\frac{1}{2-q}}<\Lambda$. By contradiction, suppose that there exists $u_{0} \in \mathscr{N}^{0}$. Obviously, $u_{0} \neq 0$ and $u_{0} \in \mathscr{N}$, it follows that

$$
\left\|u_{0}\right\|_{\mu}^{2}=\int_{\Omega} f(x)\left|u_{0}\right|^{p} d x+\int_{\Omega} g(x)\left|u_{0}\right|^{q} d x
$$

and

$$
(p-2)\left\|u_{0}\right\|_{\mu}^{2}=(p-q) \int_{\Omega} g(x)\left|u_{0}\right|^{q} d x
$$

From (2.7) and (2.8), one has

$$
\int_{\Omega} f(x)\left|u_{0}\right|^{p} d x=\frac{2-q}{p-q}\left\|u_{0}\right\|_{\mu}^{2}
$$


Then, according to (2.4) and (2.9), for $|f|_{\frac{2^{*}}{2^{*}-p}}^{\frac{1}{p-2}}|g|_{\frac{2^{*}}{2^{*}-q}}^{\frac{1}{2-q}}<\Lambda$, we have

$$
\begin{aligned}
0< & \frac{2-q}{p-2}\left(\frac{p-2}{p-q}\right)^{\frac{p-q}{2-q}} \frac{\left\|u_{0}\right\|_{\mu}^{\frac{2(p-q)}{2-q}}}{\left(\int_{\Omega} g(x)\left|u_{0}\right|^{q} d x\right)^{\frac{p-2}{2-q}}} \\
& -\int_{\Omega} f(x)\left|u_{0}\right|^{p} d x \\
= & \frac{2-q}{p-2}\left(\frac{p-2}{p-q}\right)^{\frac{p-q}{2-q}} \frac{\left\|u_{0}\right\|_{\mu}^{\frac{2(p-q)}{2-q}}}{\left(\frac{p-2}{p-q}\right)^{\frac{p-2}{2-q}}\left\|u_{0}\right\|_{\mu}^{\frac{2(p-2)}{2-q}}}-\frac{2-q}{p-q}\left\|u_{0}\right\|_{\mu}^{2} \\
= & \frac{2-q}{p-2}\left\|u_{0}\right\|_{\mu}^{2}-\frac{2-q}{p-q}\left\|u_{0}\right\|_{\mu}^{2} \\
= & 0,
\end{aligned}
$$

which is a contradiction. Thus $\mathscr{N}^{0}=\{0\}$ for $|f|_{\frac{2^{*}-p}{2^{*}-p}}^{\frac{1}{p-2}}|g|_{\frac{2^{*}}{2^{*}-q}}^{\frac{1}{2-q}}<\Lambda$.

Finally, we claim that $\mathscr{N}^{-}$is a closed set for $|f|_{\frac{2^{*}-p}{2^{*}-p}}^{\frac{1}{p-2}}|g|_{\frac{2^{*}-q}{2-q}}^{\frac{1}{2 *}}<\Lambda$.

Suppose that $\left\{u_{n}\right\} \subset \mathscr{N}^{-}$such that $u_{n} \rightarrow u$ as $n \rightarrow \infty$ in $H_{0}^{1}(\Omega)$, we need prove $u \in \mathscr{N}^{-}$for $|f|_{\frac{2^{*}}{2^{*}-p}}^{\frac{1}{p-2}}|g|_{\frac{2^{*}}{2^{*}-q}}^{\frac{1}{2-q}}<\Lambda$.

Since $u_{n} \in \mathscr{N}^{-}$, one has

$$
\left\|u_{n}\right\|_{\mu}^{2}-\int_{\Omega} f(x)\left|u_{n}\right|^{p} d x-\int_{\Omega} g(x)\left|u_{n}\right|^{q} d x=0
$$

and

$$
(2-q)\left\|u_{n}\right\|_{\mu}^{2}-(p-q) \int_{\Omega} f(x)\left|u_{n}\right|^{p} d x<0 .
$$

Since $u_{n} \rightarrow u$ in $H_{0}^{1}(\Omega)$ as $n \rightarrow \infty$, it follows that

$$
\|u\|_{\mu}^{2}-\int_{\Omega} f(x)|u|^{p} d x-\int_{\Omega} g(x)|u|^{q} d x=0
$$

and

$$
(2-q)\|u\|_{\mu}^{2}-(p-q) \int_{\Omega} f(x)|u|^{p} d x \leq 0
$$

thus $u \in \mathscr{N}^{-} \cup \mathscr{N}^{0}$. If $u \in \mathscr{N}^{0}$, since $\mathscr{N}^{0}=\{0\}$ for $|f|_{\frac{2 *}{2^{*}-p}}^{\frac{1}{p-2}}|g|_{\frac{2^{*}}{2^{*}-q}}^{\frac{1}{2-q}}<\Lambda$, one has $u=0$. However, from (2.10) and (2.11), for all $u_{n} \in \mathscr{N}^{-}$, we obtain

$$
(2-q)\left\|u_{n}\right\|_{\mu}^{2}<(p-q) \int_{\Omega} f(x)\left|u_{n}\right|^{p} d x
$$


consequently, by the Hölder inequality and (1.2), one has

$$
\left\|u_{n}\right\|_{\mu}>\left[\frac{(2-q) S_{\mu}^{\frac{p}{2}}}{(p-q)|f|_{\frac{2^{*}}{2^{*}-p}}}\right]^{\frac{1}{p-2}}>0, \quad \forall u_{n} \in \mathscr{N}^{-}
$$

which contradicts $u=0$. Thus $u \in \mathscr{N}^{-}$for $|f|_{\frac{2 *}{2^{*}-p}}^{\frac{1}{p-2}}|g|_{\frac{2^{*}}{2^{*}-q}}^{\frac{1}{2-q}}<\Lambda$. Thus our claim is proved to be true.

Case $B$ : For any $u \in H_{0}^{1}(\Omega)$ such that $\int_{\Omega} f(x)|u|^{p} d x>0$, we define $\Psi \in C\left(\mathbb{R}^{+}, \mathbb{R}\right)$ by

$$
\Psi(t)=t^{2-q}\|u\|_{\mu}^{2}-t^{p-q} \int_{\Omega} f(x)|u|^{p} d x
$$

Then

$$
\Psi^{\prime}(t)=t^{1-q}\left[(2-q)\|u\|_{\mu}^{2}-(p-q) t^{p-2} \int_{\Omega} f(x)|u|^{p} d x\right],
$$

let $\Psi^{\prime}(t)=0$, one has

$$
\tilde{t}_{\text {max }}=\left[\frac{(2-q)\|u\|_{\mu}^{2}}{(p-q) \int_{\Omega} f(x)|u|^{p} d x}\right]^{\frac{1}{p-2}} .
$$

Clearly, $\Psi^{\prime}(t)>0$ for all $0<t<\tilde{t}_{\max }$ and $\Psi^{\prime}(t)<0$ for all $t>\tilde{t}_{\text {max }}$. Thus $\Psi$ achieves its maximum at $\tilde{t}_{\max }$, that is

$$
\Psi\left(\tilde{t}_{\text {max }}\right)=\frac{p-2}{2-q}\left(\frac{2-q}{p-q}\right)^{\frac{p-q}{p-2}} \frac{\|u\|_{\mu}^{\frac{2(p-q)}{p-2}}}{\left(\int_{\Omega} f(x)|u|^{p} d x\right)^{\frac{2-q}{p-2}}} .
$$

Then from (2.1)-(2.3), one gets

$$
\begin{aligned}
\Psi\left(\tilde{t}_{\text {max }}\right)-\int_{\Omega} g(x)|u|^{q} d x \geq & \frac{p-2}{2-q}\left(\frac{2-q}{p-q}\right)^{\frac{p-q}{p-2}} \frac{\|u\|_{\mu}^{\frac{2(p-q)}{p-2}}}{\left(|u|_{2^{*}}^{p}|f|_{\frac{2^{*}}{2^{*}-p}}\right)^{\frac{2-q}{p-2}}} \\
& -|g|_{\frac{2^{*}}{2^{*}-q}}|u|_{2^{*}}^{q} \\
= & \left\{\frac{p-2}{2-q}\left(\frac{2-q}{p-q}\right)^{\frac{p-q}{p-2}}\left(\frac{\|u\|_{\mu}^{2}}{|u|_{2^{*}}^{2}}\right)^{\frac{p-q}{p-2}} \frac{1}{|g|_{\frac{2^{*}}{2^{*}-q}}|f|_{\frac{2^{*}}{2^{*}-p}}^{\frac{2-q}{p-2}}}\right. \\
> & -1\}|g|_{\frac{2^{*}}{2^{*}-q}}|u|_{2^{*}}^{q}
\end{aligned}
$$

for all $|f|_{\frac{2^{*}}{2^{*}-p}}^{\frac{1}{p-2}}|g|_{\frac{2^{*}}{2^{*}-q}}^{\frac{1}{2-q}}<\Lambda$, where $\Lambda$ is defined in Case A. If $\int_{\Omega} g(x)|u|^{q} d x \leq 0$, since $\Psi(0)=0$ and $\Psi(t) \rightarrow-\infty$ as $t \rightarrow+\infty$, there exists a unique $t^{-}$with $t^{-}>\tilde{t}_{\max }$ such that

$$
\Psi\left(t^{-}\right)=\int_{\Omega} g(x)|u|^{q} d x, \quad \Psi^{\prime}\left(t^{-}\right)<0,
$$


thus it follows that $t^{-} u \in \mathscr{N}^{-}$. Moreover from (2.5) and

$$
\frac{d I(t u)}{d t}=t^{q-1}\left[\Psi(t)-\int_{\Omega} g(x)|u|^{q} d x\right]
$$

consequently, $\frac{d I(t u)}{d t}>0$ for all $0<t<t^{-}$and $\frac{d I(t u)}{d t}<0$ for all $t>t^{-}$. Then

$$
I\left(t^{-} u\right)=\sup _{t \geq 0} I(t u)
$$

If $\int_{\Omega} g(x)|u|^{q} d x>0$, there exist $t_{0}^{+}$and $t_{0}^{-}$satisfying $0<t_{0}^{+}<\tilde{t}_{\max }<t_{0}^{-}$such that

$$
\Psi\left(t_{0}^{+}\right)=\int_{\Omega} g(x)|u|^{q} d x=\Psi\left(t_{0}^{-}\right)
$$

and

$$
\Psi^{\prime}\left(t_{0}^{+}\right)>0, \quad \Psi^{\prime}\left(t_{0}^{-}\right)<0
$$

Thus $t_{0}^{+} u \in \mathscr{N}^{+}$and $t_{0}^{-} u \in \mathscr{N}^{-}$. Moreover, according to (2.5) and (2.13), we have $\frac{d I(t u)}{d t}<0$ for all $0 \leq t<t_{0}^{+}$and $\frac{d I(t u)}{d t}>0$ for all $t_{0}^{+}<t<t_{0}^{-}$, and $\frac{d I(t u)}{d t}<0$ for all $t>t_{0}^{-}$. Consequently, one has

$$
I\left(t_{0}^{+} u\right)=\inf _{0 \leq t<\tilde{t}_{\max }} I(t u), \quad I\left(t_{0}^{-} u\right)=\sup _{t \geq \tilde{t}_{\max }} I(t u)
$$

Thus $\mathscr{N}^{ \pm}$are non-empty for all $|f|_{\frac{2^{*}}{2^{*}-p}}^{\frac{1}{p-2}}|g|_{\frac{2 *}{2^{*}-q}}^{\frac{1}{2-q}}<\Lambda$.

Next, similar to Case A, we can prove that $\mathscr{N}^{0}=\{0\}$ and $\mathscr{N}^{-}$is a closed set for $|f|_{\frac{2^{*}}{2^{*}-p}}^{\frac{1}{p-2}}|g|_{\frac{2^{*}-q}{2^{*}-q}}^{\frac{1}{2-q}}<$ $\Lambda$. We omit the detail proof. Thus the proof of Lemma 2.1 is completed.

Lemma 2.2 - Given $u \in \mathscr{N}^{+}\left(\mathscr{N}^{-}\right)$and for all $w \in H_{0}^{1}(\Omega)$, then there exist $\varepsilon>0$ and a continuous differentiable function $t=t(w)>0,\|w\|_{\mu}<\varepsilon$ satisfying that

$$
t(0)=1, \quad t(w)(u+w) \in \mathscr{N}^{+}\left(\mathscr{N}^{-}\right), \quad \forall w \in H_{0}^{1}(\Omega)\|w\|_{\mu}<\varepsilon .
$$

Proof : For all $u \in \mathscr{N}^{+}$, define $F: \mathbb{R} \times H_{0}^{1}(\Omega) \rightarrow \mathbb{R}$ by

$$
F(t, w)=t^{2-p}\|u+w\|_{\mu}^{2}-t^{q-p} \int_{\Omega} g(x)|u+w|^{q} d x-\int_{\Omega} f(x)|u+w|^{p} d x,
$$

then

$$
F_{t}(t, w)=(2-p) t^{1-p}\|u+w\|_{\mu}^{2}-(q-p) t^{q-p-1} \int_{\Omega} g(x)|u+w|^{q} d x .
$$

Since $u \in \mathscr{N}^{+}$, it follows that $F(1,0)=0$ and

$$
F_{t}(1,0)=(2-p)\|u\|_{\mu}^{2}+(p-q) \int_{\Omega} g(x)|u|^{q} d x>0 .
$$


Thus, applying the implicit function theorem at the point $(1,0)$, we can obtain $\varepsilon>0$ and a continuous differentiable $t: B(0, \varepsilon) \subset H_{0}^{1}(\Omega) \rightarrow \mathbb{R}$ satisfying that

$$
t(0)=1, \quad t(w)>0, \quad t(w)(u+w) \in \mathscr{N}^{+}, \quad \forall w \in H_{0}^{1}(\Omega) \text { with }\|\omega\|_{\mu}<\varepsilon .
$$

Similarly, we can prove that the conclusion of the case $u \in \mathscr{N}^{-}$is true. This completes the proof of Lemma 2.2.

Lemma $2.3-I$ is coercive and bounded from below on $\mathscr{N}$.

PROOF : From (2.2) and (2.3), by the Sobolev inequality and (1.2), one has

$$
\begin{aligned}
& \int_{\Omega} f(x)|u|^{p} d x \leq S_{\mu}^{-\frac{p}{2}}|f|_{\frac{2^{*}}{2^{*}-p}}\|u\|_{\mu}^{p}, \\
& \int_{\Omega} g(x)|u|^{q} d x \leq S_{\mu}^{-\frac{q}{2}}|g|_{\frac{2^{*}}{2^{*}-q}}\|u\|_{\mu}^{q} .
\end{aligned}
$$

Consequently, for all $u \in \mathscr{N}$, it follows that

$$
\begin{aligned}
I(u) & =\frac{1}{2}\|u\|_{\mu}^{2}-\frac{1}{p} \int_{\Omega} f(x)|u|^{p} d x-\frac{1}{q} \int_{\Omega} g(x)|u|^{q} d x \\
& =\left(\frac{1}{2}-\frac{1}{p}\right)\|u\|_{\mu}^{2}-\left(\frac{1}{q}-\frac{1}{p}\right) \int_{\Omega} g(x)|u|^{q} d x \\
& \geq\left(\frac{1}{2}-\frac{1}{p}\right)\|u\|_{\mu}^{2}-\left(\frac{1}{q}-\frac{1}{p}\right) S_{\mu}^{-\frac{q}{2}}|g|_{\frac{2^{*}}{2^{*}-q}}\|u\|_{\mu}^{p},
\end{aligned}
$$

this implies that $I$ is coercive and bounded from below on $\mathscr{N}$. Then the proof of Lemma 2.3 is completed.

\section{PROOF OF THEOREMS}

From Lemma 2.1 and Lemma 2.3, for $|f|_{\frac{2^{*}}{2^{*}-p}}^{\frac{1}{p-2}}|g|_{\frac{2^{*}-q}{2^{*}-q}}^{\frac{1}{2-q}}<\Lambda$, the following definitions are well defined

$$
m^{+}=\inf _{u \in \mathscr{N}^{+}} I(u), \quad m^{-}=\inf _{u \in \mathscr{N}^{-}} I(u) .
$$

Moreover, we can claim that $m^{+}<0$. In fact, for all $u \in \mathscr{N}^{+}$, we have

$$
(p-q) \int_{\Omega} g(x)|u|^{q} d x>(p-2)\|u\|_{\mu}^{2},
$$

consequently, since $2<p<2^{*}, 1<q<2$ and $u \neq 0$, it follows that

$$
\begin{aligned}
I(u) & =\frac{1}{2}\|u\|_{\mu}^{2}-\frac{1}{p} \int_{\Omega} f(x)|u|^{p} d x-\frac{1}{q} \int_{\Omega} g(x)|u|^{q} d x \\
& =\left(\frac{1}{2}-\frac{1}{p}\right)\|u\|_{\mu}^{2}-\left(\frac{1}{q}-\frac{1}{p}\right) \int_{\Omega} g(x)|u|^{q} d x \\
& <\frac{p-2}{2 p}\|u\|_{\mu}^{2}-\frac{p-2}{p q}\|u\|_{\mu}^{2} \\
& <0
\end{aligned}
$$


this implies that $m^{+}<0$. In this part, we prove that problem (1.1) has a nonnegative local minimizer solution in $\mathscr{N}^{+}$and $\mathscr{N}^{-}$, respectively. Now, we give the proof of Theorem 1.1. into two steps.

Proof OF THEOREM 1.1 : Let $|f|_{\frac{2^{*}}{2^{*}-p}}^{\frac{1}{p-2}}|g|_{\frac{2^{*}}{2^{*}-q}}^{\frac{1}{2-q}}<\Lambda$. The proof of Theorem 1.1 will be divided

Step 1 : We prove that there exists a nonnegative solution of problem (1.1) in $\mathscr{N}^{+}$.

Obviously, $\mathscr{N}^{+} \cup \mathscr{N}^{0}$ is a closed set for $|f|_{\frac{2^{*}-p}{2^{*}-p}}^{\frac{1}{p-2}}|g|_{\frac{2^{*}}{2^{*}-q}}^{\frac{1}{2-q}}<\Lambda$. From Lemma 2.3, $\inf _{u \in \mathscr{N}+\cup \mathscr{N}^{0}} I(u)$ is well defined. Applying Ekeland's variational principle (see [13]) to this minimization problem, there exists a sequence $\left\{u_{n}\right\} \subset \mathscr{N}^{+} \cup \mathscr{N}^{0}$ with the following properties:

(i) $I\left(u_{n}\right)<\inf _{u \in \mathscr{N}+\cup \mathscr{N}^{0}} I(u)+\frac{1}{n}$;

(ii) $I(u) \geq I\left(u_{n}\right)-\frac{1}{n}\left\|u-u_{n}\right\|_{\mu}, \quad \forall u \in \mathscr{N}^{+} \cup \mathscr{N}^{0}$.

Since $I(u)=I(|u|)$, we can assume that $u_{n}(x) \geq 0$ for all $x \in \Omega$. Obviously, $\left\{u_{n}\right\}$ is bounded in $H_{0}^{1}(\Omega)$, going if necessary to a subsequence, still denoted by $\left\{u_{n}\right\}$, there exists $u_{*} \geq 0$ such that

$$
\left\{\begin{array}{l}
u_{n} \rightarrow u_{*}, \quad \text { weakly in } \mathrm{H}_{0}^{1}(\Omega), \\
u_{n} \rightarrow u_{*}, \quad \text { strongly in } \mathrm{L}^{\mathrm{s}}(\Omega), 1 \leq \mathrm{s}<2^{*}, \\
u_{n}(x) \rightarrow u_{*}(x), \quad \text { a.e. in } \Omega,
\end{array}\right.
$$

as $n \rightarrow \infty$. Now we will prove that $u_{*}$ is a nonnegative solution of problem (1.1).

Firstly, we prove that $u_{*}(x) \not \equiv 0$ in $\Omega$. By the Vitali theorem (see [25] pp: 133), we claim that

$$
\lim _{n \rightarrow \infty} \int_{\Omega} f(x)\left|u_{n}\right|^{p} d x=\int_{\Omega} f(x)\left|u_{*}\right|^{p} d x
$$

Indeed, we only need prove that $\left\{\int_{\Omega} f(x)\left|u_{n}\right|^{p} d x, n \in N\right\}$ is equi-absolutely-continuous. Note that $\left\{u_{n}\right\}$ is bounded in $H_{0}^{1}(\Omega)$, by the Sobolev embedding theorem, then exists a constant $C_{1}>0$ such that $\left|u_{n}\right|_{2^{*}} \leq C_{1}<\infty$. From (2.2), for every $\varepsilon>0$, setting $\delta>0$, when $E \subset \Omega$ with mes $E<\delta$, we have

$$
\begin{aligned}
\int_{E} f(x)\left|u_{n}\right|^{p} d x & \leq\left|u_{n}\right|_{2^{*}}^{p}\left(\int_{E}|f|^{\frac{2^{*}}{2^{*}-p}} d x\right)^{\frac{2^{*}-p}{2^{*}}} \\
& <\varepsilon
\end{aligned}
$$

where the last inequality is from the absolutely-continuity of $\int_{\Omega}|f|^{\frac{2^{*}}{2^{*}-p}} d x$. Thus, our claim is true. Similarly,

$$
\lim _{n \rightarrow \infty} \int_{\Omega} g(x)\left|u_{n}\right|^{q} d x=\int_{\Omega} g(x)\left|u_{*}\right|^{q} d x
$$


By the weakly lower semicontinuity of the norm, combining (3.1) and (3.2), we have

$$
\begin{aligned}
I\left(u_{*}\right) & =\frac{1}{2}\left\|u_{*}\right\|_{\mu}^{2}-\frac{1}{p} \int_{\Omega} f(x)\left|u_{*}\right|^{p} d x-\frac{1}{q} \int_{\Omega} g(x)\left|u_{*}\right|{ }^{q} d x \\
& \leq \liminf _{n \rightarrow \infty}\left[\frac{1}{2}\left\|u_{n}\right\|_{\mu}^{2}-\frac{1}{p} \int_{\Omega} f(x)\left|u_{n}\right|^{p} d x-\frac{1}{q} \int_{\Omega} g(x)\left|u_{n}\right|^{q} d x\right] \\
& =\liminf _{n \rightarrow \infty} I\left(u_{n}\right) \\
& =m^{+} \\
& <0
\end{aligned}
$$

which implies that $u_{*}(x) \not \equiv 0$ in $\Omega$. Thus $u_{*} \geq 0, u_{*} \not \equiv 0$ in $\Omega$. Since

$$
m^{+}=\inf _{u \in \mathscr{N}+} I(u)<, 0
$$

thus

$$
\inf _{u \in \mathscr{N}^{+} \cup \mathscr{N}^{0}} I(u)=\inf _{u \in \mathscr{N}^{+}} I(u)<0
$$

Moreover, combining $(i)$ with (3.3), for all $n$ large enough we have $I\left(u_{n}\right)<0$. Therefore, the sequence $\left\{u_{n}\right\}$ has a subsequence, still denoted by $\left\{u_{n}\right\}$, such that $\left\{u_{n}\right\} \subset \mathscr{N}^{+}$.

Secondly, we prove that $u_{*}$ is a weak solution of problem (1.1). Let $s>0$ small enough, $\varphi \in$ $H_{0}^{1}(\Omega)$, we choose $u=u_{n}, w=s \varphi \in H_{0}^{1}(\Omega)$ in Lemma 2.2, thus we get $t_{n}(s)=t_{n}(s \varphi)$ satisfying

$$
t_{n}(0)=1, \quad t_{n}(s)\left(u_{n}+s \varphi\right) \in \mathscr{N}^{+}
$$

Note that

$$
\left\|u_{n}\right\|_{\mu}^{2}-\int_{\Omega} f(x) u_{n}^{p} d x-\int_{\Omega} g(x) u_{n}^{q} d x=0
$$

Then, from (ii), one has

$$
\begin{aligned}
\frac{\left|t_{n}(s)-1\right| \cdot\left\|u_{n}\right\|_{\mu}+s t_{n}(s)\|\varphi\|_{\mu} \geq}{n} \geq & \frac{1}{n}\left\|t_{n}(s)\left(u_{n}+s \varphi\right)-u_{n}\right\|_{\mu} \\
\geq & I\left(u_{n}\right)-I\left[t_{n}(s)\left(u_{n}+s \varphi\right)\right], \\
= & \frac{1-t_{n}^{2}(s)}{2}\left\|u_{n}\right\|_{\mu}^{2}+\frac{t_{n}^{p}(s)-1}{p} \int_{\Omega} f(x)\left|u_{n}+s \varphi\right|^{p} d x \\
& +\frac{t_{n}^{q}(s)-1}{q} \int_{\Omega} g(x)\left|u_{n}+s \varphi\right|^{q} d x \\
& +\frac{t_{n}^{2}(s)}{2}\left(\left\|u_{n}\right\|_{\mu}^{2}-\left\|u_{n}+s \varphi\right\|_{\mu}^{2}\right) \\
& +\frac{1}{p} \int_{\Omega} f(x)\left(\left|u_{n}+s \varphi\right|^{p}-u_{n}^{p}\right) d x \\
& +\frac{1}{q} \int_{\Omega} g(x)\left(\left|u_{n}+s \varphi\right|^{q}-\left|u_{n}\right|^{q}\right) d x .
\end{aligned}
$$


Consequently, combining with (3.4), dividing by $s$ and letting $s \rightarrow 0^{+}$, it follows that

$$
\begin{aligned}
\frac{\left|t_{n}^{\prime}(0)\right|\left\|u_{n}\right\|_{\mu}+\|\varphi\|_{\mu}}{n} \geq & -t_{n}^{\prime}(0)\left\|u_{n}\right\|_{\mu}^{2}+t_{n}^{\prime}(0) \int_{\Omega} f(x)\left|u_{n}\right|^{p} d x \\
& +t_{n}^{\prime}(0) \int_{\Omega} g(x)\left|u_{n}\right|^{q} d x-\int_{\Omega}\left[\left(\nabla u_{n}, \nabla \varphi\right)-\frac{\mu}{|x|^{2}} u_{n} \varphi\right] d x \\
& +\int_{\Omega} f(x)\left|u_{n}\right|^{p-2} u_{n} \varphi d x+\int_{\Omega} g(x)\left|u_{n}\right|^{q-2} u_{n} \varphi d x \\
= & -t_{n}^{\prime}(0)\left(\left\|u_{n}\right\|_{\mu}^{2}-\int_{\Omega} f(x)\left|u_{n}\right|^{p} d x-\int_{\Omega} g(x)\left|u_{n}\right|^{q} d x\right) \\
& -\int_{\Omega}\left[\left(\nabla u_{n}, \nabla \varphi\right)-\frac{\mu}{|x|^{2}} u_{n} \varphi\right] d x \\
& +\int_{\Omega} f(x)\left|u_{n}\right|^{p-2} u_{n} \varphi d x+\int_{\Omega} g(x)\left|u_{n}\right|^{q-2} u_{n} \varphi d x \\
= & -\int_{\Omega}\left[\left(\nabla u_{n}, \nabla \varphi\right)-\frac{\mu}{|x|^{2}} u_{n} \varphi\right] d x \\
& +\int_{\Omega} f(x)\left|u_{n}\right|^{p-2} u_{n} \varphi d x+\int_{\Omega} g(x)\left|u_{n}\right|^{q-2} u_{n} \varphi d x .
\end{aligned}
$$

Hence, we can deduce

$$
\begin{aligned}
\frac{\left|t_{n}^{\prime}(0)\right|\left\|u_{n}\right\|_{\mu}+\|\varphi\|_{\mu}}{n} \leq & \int_{\Omega}\left[\left(\nabla u_{n}, \nabla \varphi\right)-\frac{\mu}{|x|^{2}} u_{n} \varphi\right] d x \\
& -\int_{\Omega} f(x)\left|u_{n}\right|^{p-2} u_{n} \varphi d x-\int_{\Omega} g(x)\left|u_{n}\right|^{q-2} u_{n} \varphi d x
\end{aligned}
$$

for any $\varphi \in H_{0}^{1}(\Omega)$. Since (3.5) also holds for $-\varphi$, one gets

$$
\begin{aligned}
\frac{\left|t_{n}^{\prime}(0)\right|\left\|u_{n}\right\|_{\mu}+\|\varphi\|_{\mu}}{n}= & \int_{\Omega}\left[\left(\nabla u_{n}, \nabla \varphi\right)-\frac{\mu}{|x|^{2}} u_{n} \varphi\right] d x \\
& -\int_{\Omega} f(x)\left|u_{n}\right|^{p-2} u_{n} \varphi d x-\int_{\Omega} g(x)\left|u_{n}\right|^{q-2} u_{n} \varphi d x .
\end{aligned}
$$

Moreover, Lemma 2.2 suggests that there exists a constant $C_{2}>0$, such that $\left|t_{n}^{\prime}(0)\right| \leq C_{2}$ for all $n \in \mathbf{N}^{+}$. Therefore, from the boundedness of $\left\{u_{n}\right\}$, we can choose a subsequence of $\left\{u_{n}\right\}$ still denoted by $\left\{u_{n}\right\}$, passing to the limit as $n \rightarrow \infty$ in (3.6), we get

$$
\int_{\Omega}\left[\left(\nabla u_{*}, \nabla \varphi\right)-\frac{\mu}{|x|^{2}} u_{*} \varphi\right] d x-\int_{\Omega} f(x)\left|u_{*}\right|^{p-2} u_{*} \varphi d x-\int_{\Omega} g(x)\left|u_{*}\right|^{q-2} u_{*} \varphi d x=0,
$$

for all $\varphi \in H_{0}^{1}(\Omega)$, this implies that $u_{*}$ is a weak solution of problem (1.1).

Finally, we prove that $u_{*} \in \mathscr{N}^{+}$. Choosing $\varphi=u_{*}$ in (3.7), one has

$$
\left\|u_{*}\right\|_{\mu}^{2}-\int_{\Omega} f(x)\left|u_{*}\right|^{p} d x-\int_{\Omega} g(x)\left|u_{*}\right|^{q} d x=0,
$$


this implies $u_{*} \in \mathscr{N}$. Since $u_{n} \in \mathscr{N}^{+}$, we have

$$
(2-p)\left\|u_{n}\right\|_{\mu}^{2}+(p-q) \int_{\Omega} g(x)\left|u_{n}\right|^{q} d x>0 .
$$

Consequently, one has

$$
(2-p)\left\|u_{*}\right\|_{\mu}^{2}+(p-q) \int_{\Omega} g(x)\left|u_{*}\right|^{q} d x \geq 0 .
$$

We claim that

$$
(2-p)\left\|u_{*}\right\|_{\mu}^{2}+(p-q) \int_{\Omega} g(x)\left|u_{*}\right|^{q} d x>0 .
$$

In fact, suppose

$$
(2-p)\left\|u_{*}\right\|_{\mu}^{2}+(p-q) \int_{\Omega} g(x)\left|u_{*}\right|^{q} d x=0,
$$

according to Lemma 2.1, $\mathscr{N}^{0}=\{0\}$ for $|f|_{\frac{2^{*}-p}{2^{*}-p}}^{\frac{1}{p-2}}|g|_{\frac{2^{*}}{2^{*}-q}}^{\frac{1}{2-q}}<\Lambda$, then one has $u_{*} \equiv 0$ in $\Omega$. This contradicts $u_{*} \not \equiv 0$ in $\Omega$. Thus our claim is true. Therefore, $u_{*} \in \mathscr{N}^{+}$.

Step 2 : We prove that there exists a nonnegative solution of problem (1.1) in $\mathscr{N}^{-}$.

Similarly, applying Ekeland's variational principle to the minimization problem $m^{-}=\inf _{u \in \mathscr{N}^{-}} I(u)$, there exists a sequence $\left\{v_{n}\right\} \subset \mathscr{N}^{-}$with the following properties:

(i) $I\left(v_{n}\right)<m^{-}+\frac{1}{n}$;

(ii) $I(u) \geq I\left(v_{n}\right)-\frac{1}{n}\left\|u-v_{n}\right\|_{\mu}, \forall u \in \mathscr{N}^{-}$.

Since $I(u)=I(|u|)$, we can assume from the beginning that $v_{n}(x) \geq 0$ for all $x \in \Omega$. Obviously, $\left\{v_{n}\right\}$ is bounded in $H_{0}^{1}(\Omega)$, going if necessary to a subsequence, still denoted by $\left\{v_{n}\right\}$, there exists $u_{* *} \geq 0$ such that

$$
\left\{\begin{array}{l}
v_{n} \rightarrow u_{* *}, \quad \text { weakly in } \mathrm{H}_{0}^{1}(\Omega), \\
v_{n} \rightarrow u_{* *}, \quad \text { strongly in } \mathrm{L}^{\mathrm{s}}(\Omega), 1 \leq \mathrm{s}<2^{*}, \\
v_{n}(x) \rightarrow u_{* *}(x), \quad \text { a.e. in } \Omega,
\end{array}\right.
$$

as $n \rightarrow \infty$. Now we will prove that $u_{* *}$ is a nonnegative solution of problem (1.1).

Firstly, we prove that $u_{* *}(x) \not \equiv 0$ in $\Omega$. Since $v_{n} \in \mathscr{N}^{-}$, we have

$$
(p-2)\left\|v_{n}\right\|_{\mu}^{2}>(p-q) \int_{\Omega} g(x)\left|v_{n}\right|^{q} d x,
$$

which is equivalent to

$$
(2-q)\left\|v_{n}\right\|_{\mu}^{2}<(p-q) \int_{\Omega} f(x)\left|v_{n}\right|^{p} d x
$$


Consequently, combining with (2.2) and (2.1), one has

$$
\begin{aligned}
(2-q)\left\|v_{n}\right\|_{\mu}^{2} & \leq(p-q) \int_{\Omega} f(x)\left|v_{n}\right|^{p} d x \\
& \leq(p-q)|f|_{\frac{2^{*}}{2^{*}-p}}\left|v_{n}\right|_{2^{*}}^{p} \\
& <(p-q)|f|_{\frac{2^{*}}{2^{*}-p}} S_{\mu}^{-\frac{p}{2}}\left\|v_{n}\right\|_{\mu}^{p}
\end{aligned}
$$

thus

$$
\left\|v_{n}\right\|_{\mu}>\left[\frac{(2-q) S_{\mu}^{\frac{p}{2}}}{(p-q)|f|_{\frac{2^{*}}{2^{*}-p}}}\right]^{\frac{1}{p-2}}, \quad \forall v_{n} \in \mathscr{N}^{-},
$$

which implies $u_{* *} \geq 0$ and $u_{* *} \not \equiv 0$.

Secondly, we prove that $u_{* *}$ is a weak solution of problem (1.1). According to Lemma 2.2, we can word by word repeat the corresponding arguments in Step 1 , and obtain that $u_{* *}$ is a nonnegative solution of problem (1.1), that is,

$$
\int_{\Omega}\left[\left(\nabla u_{* *}, \nabla \varphi\right)-\frac{\mu}{|x|^{2}} u_{* *} \varphi\right] d x-\int_{\Omega} f(x)\left|u_{* *}\right|^{p-2} u_{* *} \varphi d x-\int_{\Omega} g(x)\left|u_{* *}\right|^{q-2} u_{* *} \varphi d x=0,
$$

for all $\varphi \in H_{0}^{1}(\Omega)$.

Finally, we prove that $u_{* *} \in \mathscr{N}^{-}$. Choosing $\varphi=u_{* *}$ in above equality, we have

$$
\left\|u_{* *}\right\|_{\mu}^{2}-\int_{\Omega} f(x)\left|u_{* *}\right|^{p} d x-\int_{\Omega} g(x)\left|u_{* *}\right|^{q} d x=0,
$$

this implies $u_{* *} \in \mathscr{N}$. Since $v_{n} \in \mathscr{N}^{-}$, we have

$$
(2-p)\left\|v_{n}\right\|_{\mu}^{2}+(p-q) \int_{\Omega} g(x)\left|v_{n}\right|^{q} d x<0 .
$$

Consequently, one has

$$
(2-p)\left\|u_{* *}\right\|_{\mu}^{2}+(p-q) \int_{\Omega} g(x)\left|u_{* *}\right|^{q} d x \leq 0 .
$$

We claim that

$$
(2-p)\left\|u_{* *}\right\|_{\mu}^{2}+(p-q) \int_{\Omega} g(x)\left|u_{* *}\right|^{q} d x<0
$$

In fact, suppose

$$
(2-p)\left\|u_{* *}\right\|_{\mu}^{2}+(p-q) \int_{\Omega} g(x)\left|u_{* *}\right|^{q} d x=0
$$

according to Lemma 2.1, one has $u_{* *} \equiv 0$ in $\Omega$ for $|f|_{\frac{2^{*}}{2^{*}-p}}^{\frac{1}{p-2}}|g|_{\frac{2^{*}}{2^{*}-q}}^{\frac{1}{2-q}}<\Lambda$, which contradicts $u_{* *} \not \equiv 0$ in $\Omega$. Thus our claim is true. Therefore, $u_{* *} \in \mathscr{N}^{-}$. Obviously, one has $I\left(u_{*}\right)=m^{+}$and $I\left(u_{* *}\right)=$ 
$m^{-}$. Thus, $u_{*}$ or $u_{* *}$ is a ground state solution of problem (1.1). Then the proof of Theorem 1.1 is completed.

ProOF OF TheOREM 1.2 : According to the proof of Theorem 1.1, we only need prove that $u_{*}, u_{* *}>0$ in $\Omega$. Assume that $\left(H_{1}\right)$ holds. Since $u_{*}, u_{* *} \in H_{0}^{1}(\Omega)$, by the embedding theorem we have $u_{*}, u_{* *} \in L^{2^{*}}(\Omega)$. Since $f, g \in L^{\infty}(\Omega)$, by the regularity of weak solutions, we have $u_{*}, u_{* *} \in W^{2, \frac{2^{*}}{p}}(\Omega)$. Then, by the classical bootstrap argument, one has $u_{*} \in W^{2, s}(\Omega)$ for all $1 \leq s<\infty$. Using the embedding theorem again, we have $u_{*} \in C^{1, \alpha}(\bar{\Omega})$ for some $0<\alpha<1$. Since $u_{*} \geq 0, u_{*} \not \equiv 0$ and $g(x) \geq 0, g \not \equiv 0$, one has

$$
\begin{aligned}
-\Delta u_{*} & =\frac{\mu}{|x|^{2}} u_{*}+f(x) u_{*}^{p-1}+g(x) u_{*}^{q-1} \\
& \geq f(x) u_{*}^{p-1} \\
& \geq-f^{-} u_{*}^{p-2} u_{*} \\
& \geq-C u_{*},
\end{aligned}
$$

where $f^{ \pm}=\max \{ \pm f, 0\}$ and $f=f^{+}-f^{-}$, and $C>0$ is a constant. Thus, by the strong maximum principle, one has $u_{*}>0$ in $\Omega$. Similarly, we can obtain that $u_{* *}>0$ in $\Omega$. Then the proof of Theorem 1.2 is completed.

In order to prove Theorem 1.3, we give the following lemma.

Lemma 3.1 - Given $u \in \mathscr{N}$ and for all $w \in H_{0}^{1}(\Omega)$, then there exist $\varepsilon>0$ and a continuous differentiable function $t=t(w)>0,\|w\|_{\mu}<\varepsilon$ satisfying that

$$
t(0)=1, \quad t(w)(u+w) \in \mathscr{N}, \quad \forall w \in H_{0}^{1}(\Omega)\|w\|_{\mu}<\varepsilon .
$$

PROOF : The proof is similar to the proof of Lemma 2.2, we omit the detail proof.

Now, we give the main proof of Theorem 1.3.

Proof of TheOrem 1.3 : According to Lemma 2.1 and Lemma 2.3, one obtains that $m=$ $\inf _{u \in \mathscr{N}} I(u)$ is well defined. Obviously, $m \leq m^{-}<0$ and $m \leq m^{+}$. From Lemma 3.1, by Ekeland's variational principle, similarly to Step 1 of the proof of Theorem 1.1, we can prove that there exists a nonnegative solution of problem (1.1) in $\mathscr{N}$. Without loss of generality, we also denote this solution by $u_{*}$, then $I\left(u_{*}\right)=m$ and $u_{*} \in \mathscr{N}$. Now, we claim that $u_{*} \in \mathscr{N}^{+}$. On the contrary, assume that $u_{*} \in \mathscr{N}^{-}\left(\mathscr{N}^{0}=\emptyset\right.$ for $\left.|f|_{\frac{2^{*}}{2^{*}-p}}^{\frac{1}{p-2}}|g|_{\frac{2 *^{*}}{2^{*}-q}}^{\frac{1}{2-q}}<\Lambda\right)$. From $\left(H_{2}\right)$, one has $\int_{\Omega} f(x)|u|^{p} d x>0$ and $\int_{\Omega} g(x)|u|^{q} d x>0$. By Lemma 2.1, then there exist positive numbers $t_{0}^{+}<t_{\max }<t_{0}^{-}=1$ such that $t_{0}^{+} u_{*} \in \mathscr{N}^{+}$and $t_{0}^{-} u_{*} \in \mathscr{N}^{-}$and

$$
I\left(t_{0}^{+} u_{*}\right) \leq I\left(t_{0}^{-} u_{*}\right)=I\left(u_{*}\right)=m
$$


which is a contradiction. Hence, $I\left(u_{*}\right)=m=m^{+}$and $u_{*} \in \mathscr{N}^{+}$. Obviously, $u_{*}$ is a nonnegative ground state solution of problem (1.1). Similarly to Step 2 of the proof of Theorem 1.1, we get that there exists a nonnegative solution $u_{* *} \in \mathscr{N}^{-}$.

Now, we only need prove that $u_{*}, u_{* *}>0$ in $\Omega$. Assume that $\left(H_{2}\right)$ holds, it follows from (3.7) that

$$
\int_{\Omega}\left[\left(\nabla u_{*}, \nabla \varphi\right)-\frac{\mu}{|x|^{2}} u_{*} \varphi\right] d x \geq 0 \quad \text { for } \varphi \in \mathrm{H}_{0}^{1}(\Omega),
$$

which means that $u_{*}$ satisfies

$$
-\Delta u_{*} \geq 0, \text { in } \Omega
$$

Since $u_{*} \geq 0, u_{*} \not \equiv 0$, the strong maximum principle suggests that $u_{*}>0$ in $\Omega$. Similarly, we can prove that $u_{* *}>0$ in $\Omega$. This completes the proof of Theorem 1.3.

\section{ACKNOWLEDGEMENT}

The authors would like to thank the anonymous referees and the handling editor for their careful reading. The paper is supported by the Scientific Research Fund of Sichuan Provincial Education Department (18ZA0471), the Meritocracy Research Funds of China West Normal University (17YC383), the Fundamental Research Funds of China West Normal University (17E089, 18B015, 18D052) and the Innovation Team Research Funds of China West Normal University (CXTD2018-8).

\section{REFERENCES}

1. A. Ambrosetti, H. Brézis, and G. Cerami, Combined effects of concave and convex nonlinearities in some elliptic problems, J. Funct. Anal., 122 (1994), 519-543.

2. B. Barrios, E. Colorado, R. Servadei, and F. Soria, A critical fractional equation with concave-convex power nonlinearities, Ann. Inst. H. Poincaré Anal. Non Linéaire, 32 (2015), 875-900.

3. T. Bartsch, Y. H. Ding, and C. Lee, Periodic solutions of a wave equation with concave and convex nonlinearities, J. Diff. Equ., 153 (1999), 121-141.

4. T. Bartsch and M. Willem, On an elliptic equation with concave and convex nonlinearities, Proc. Amer. Math. Soc., 123 (1995), 3555-3561.

5. L. Caffarelli, R. Kohn, and L. Nirenberg, First order interpolation inequality with weights, Compositio Math., 53 (1984), 259-275.

6. D. M. Cao, S. J. Peng, and S. S. Yan, Infinitely many solutions for $p$-Laplacian equation involving critical Sobolev growth, J. Funct. Anal., 262 (2012), 2861-2902. 
7. G. Carboni and D. Mugnai, On some fractional equations with convex-concave and logistic-type nonlinearities, J. Diff. Equ., 262 (2017), 2393-2413.

8. M. L. M. Carvalho, E. D. da Silva, and C. Goulart, Quasilinear elliptic problems with concave-convex nonlinearities, Commun. Contemp. Math., 19 (2017), 25pp.

9. J. Q. Chen, Some further results on a semilinear equation with concave-convex nonlinearity, Nonlinear Anal., 62 (2005), 71-87.

10. Y. P. Chen and J. Q. Chen, Existence of multiple positive weak solutions and estimates for extremal values for a class of concave-convex elliptic problems with an inverse-square potential, Commun. Pure Appl. Anal., 16 (2017), 1531-1552.

11. W. J. Chen and S. B. Deng, The Nehari manifold for a fractional $p$-Laplacian system involving concaveconvex nonlinearities, Nonlinear Anal. Real World Appl., 27 (2016), 80-92.

12. B. T. Cheng, Xian $\mathrm{Wu}$, and Jun Liu, Multiple solutions for a class of Kirchhoff type problems with concave nonlinearity, NoDEA Nonlinear Differential Equations Appl., 19 (2012), 521-537.

13. I. Ekeland, On the variational principle, J. Math. Anal. Appl., 47 (1974), 324-353.

14. J. Garcia-Azorero, I. Peral, and J. D. Rossi, A convex-concave problem with a nonlinear boundary condition, J. Diff. Equ., 198 (2004), 91-128.

15. N. Ghoussoub and C. Yuan, Multiple solutions for quasilinear PDEs involving the critical Sobolev and Hardy exponents, Trans. Amer. Math. Soc., 352 (2000), 5703-5743.

16. D. Gilbarg and N. S. Trudinger, Elliptic partial differential equations of second order, Springer-Verlag, Berlin (2001).

17. T. S. Hsu and H. L. Lin, Three positive solutions for semilinear elliptic problems involving concave and convex nonlinearities, Proc. Roy. Soc. Edinburgh Sect. A, 142 (2012), 115-135.

18. Y. S. Huang, Multiple positive solutions of nonhomogeneous equations involving the $p$-Laplacian, Nonlinear Anal., 43 (2001), 905-922.

19. P. Korman, On uniqueness of positive solutions for a class of semilinear equations, Discrete Contin. Dyn. Syst., 8 (2002), 865-871.

20. P. Korman and Y. Li, Exact multiplicity of positive solutions for concave-convex and convex-concave nonlinearities, J. Diff. Equ., 257 (2014), 3730-3737.

21. J. F. Liao, Y. Pu, X. F. Ke, and C. L. Tang, Multiple positive solutions for Kirchhoff type problems involving concave-convex nonlinearities, Commun. Pure Appl. Anal., 16 (2017), 2157-2175.

22. H. L. Lin, Positive solutions for nonhomogeneous elliptic equations involving critical Sobolev exponent, Nonlinear Anal., 75 (2012), 2660-2671. 
23. A. Molino and J. D. Rossi, A concave-convex problem with a variable operator, Calc. Var. Partial Differ. Equ., 57 (2018), 26pp.

24. H. Ramos Quoirin and K. Umezu, On a concave-convex elliptic problem with a nonlinear boundary condition, Ann. Mat. Pura Appl., 195 (2016), 1833-1863.

25. W. Rudin, Real and complex analysis, McGraw-Hill, New York, London etc. (1966).

26. Y. J. Sun, Estimates for extremal values of $-\Delta u=h(x) u^{q}+\lambda W(x) u^{p}$, Commun. Pure Appl. Anal., 9 (2010), 751-760.

27. Y. J. Sun and S. J. Li, A nonlinear elliptic equation with critical exponent: Estimates for extremal values, Nonlinear Anal., 69 (2008), 1856-1869.

28. M. Tang, Exact multiplicity for semilinear elliptic Dirichlet problems involving concave and convex nonlinearities, Proc. Roy. Soc. Edinburgh Sect. A, 133 (2003), 705-717.

29. S. B. Tshinanga, On multiple solutions of semilinear elliptic equation on unbounded domains with concave and convex nonlinearities, Nonlinear Anal., 28 (1997), 809-814.

30. L. Wang, Q. L. Wei, and D. S. Kang, Multiple positive solutions for $p$-Laplace elliptic equations involving concave-convex nonlinearities and a Hardy-type term, Nonlinear Anal., 74 (2011), 626-638.

31. D. L. Wu, C. Li, and P. F. Yuan, Multiplicity solutions for a slass of fractional Hamiltonian systems with concave-convex potentials, Mediterr. J. Math., 15 (2018), 1-22.

32. D. L. Wu, C. L. Tang, and X. P. Wu, Homoclinic orbits for a class of second-order Hamiltonian systems with concave-convex nonlinearities, Electron. J. Qual. Theory Differ. Equ., 6 (2018), 18 pp. 